\title{
National Achievement Award, Canadian Linguistic Association Prix national d'excellence, Association canadienne de linguistique 2020
}

\section{Dr. Johanne Catherine Paradis}

Dr. Johanne Paradis is a recognized leader in the field, with an exceptional record of research achievements. She is also a celebrated teacher and mentor, and she has a strong record of building bridges between the academic and non-academic world. Her research focus is child (second) language acquisition and bilingualism.

Dr. Paradis holds a BA and MA in Linguistics from UBC, and a PhD (Dean's Honour List) in Psychology as well as a Graduate Diploma in Education from McGill University. After her graduation in 1998, she completed a SSHRC post-doctoral fellowship at McGill, then in 2020 joined the Department of Linguistics at the University of Alberta, where she is now a Full Professor and Associate Chair, Graduate. She is also the director of the Language Acquisition Lab, which researches bilingualism in children, and an Adjunct Professor in the Department of Communication Sciences and Disorders. Dr. Paradis is the editor of Journal of Child Language and a board member for several other journals. She has served on many administrative committees, including the Program committee of the Canadian Linguistics Association. She is popular as an invited speaker and is the recipient of several research grants and awards, as well as an award for graduate supervision.

Dr. Paradis' research focuses on child language acquisition, bilingualism, and second language acquisition in children, with an emphasis on children in immigrant and refugee families. Her research is heavily cited, and stands out both in terms of output and depth, with three edited books, 16 book chapters, and more than 60 peer-reviewed papers. She has published in areas such as education and applied psycholinguistics as well as in language acquisition. Her research is of interest not only to acquisitionists, but also to clinicians such as speech-language pathologists, as well as to educators and policymakers. She has conducted research on language acquisition in French-English bilingual children, and in collaboration with colleagues, she has captured the diversity of bilingual experiences by including children from a range of first language backgrounds. In addition to typical populations, her research has examined clinical bilingual populations, especially bilingual children with Developmental Language Disorder (also known as Specific Language Impairment). This research has clinical relevance, as it elucidates the range of bilingual trajectories. More recently, she has also conducted research on bilinguals with 
Autism Spectrum Disorder. A passionate French-English bilingual speaker, her work has shifted the focus of acquisition research to include bilingual children. This is particularly relevant in an increasingly multilingual society such as Canada.

Communicating the findings and implications of her research to concerned parties beyond academia is a pivotal part of Dr. Paradis' career. She has delivered multiple workshops and seminars across Canada to teachers, school administrators, speech pathologists, and special education professionals on different matters related to bilingualism and bilingual education. In consultation with community partners, she has set up the Child English as a Second Language Resource Centre, and created parental questionnaires, such as the Alberta Language Development Questionnaire, which examines first language development, and the Alberta Language Environment Questionnaire, which looks at language use patterns in bilingual homes. In addition to this, she has taken part in several interviews in both print and radio media and has written pieces in which she advocates for an empirical approach to bilingualism in order to dispel myths and preconceived notions. Johanne is actively involved in the Coalition of Social Inclusion (Edmonton), a group that advocates for the long-term integration, social inclusion, and settlement of newcomers to Canada, as well as addressing the issue of first language maintenance. Several of her recent grant-funded projects focus on best practices and challenges for language and literacy among immigrants and refugees.

In summary, Johanne Paradis has set a stellar example of what it means to be a Canadian scholar, in terms of both pure research and knowledge mobilization. She has also excelled as a mentor and administrator. The Canadian Linguistic Association is delighted to recognize this great service by awarding Dr. Paradis our National Achievement Award for 2020.

$$
* * *
$$

Dre Johanne Paradis est une leader reconnue dans son domaine, avec un dossier de recherche exceptionnel. Elle est également une enseignante et une mentor hors pair et a un dossier solide en matière d'établissement de passerelles entre le monde universitaire et non-universitaire. Son travail de recherche se situe dans le domaine de l'acquisition de la langue seconde chez l'enfant et du bilinguisme.

Dre Paradis est titulaire d'un baccalauréat et d'une maîtrise en linguistique de l'Université de la Colombie-Britannique, ainsi que d'un doctorat (liste d'honneur du doyen) en psychologie et d'un diplôme de deuxième cycle en éducation décernés par l'Université McGill. Après avoir terminé ses études en 1998, elle a fait ses études postdoctorales à McGill avec une subvention du CRSH. Ensuite, elle s'est jointe en 2000 au Département de linguistique de l'Université de l'Alberta, où elle occupe un poste de professeur titulaire et de co-directrice au cycle supérieur. Elle est aussi la directrice du Laboratoire de l'acquisition des langues qui effectue des recherches sur le bilinguisme des enfants, et professeure adjointe au Département de sciences et troubles de la communication. Dre Paradis est également l'éditrice de la revue Journal of Child Language, et membre du conseil de plusieurs autres revues. Elle a participé à nombre de comités administratifs, dont le comité du programme de l'Association canadienne de linguistique. Elle est une conférencière invitée très 
sollicitée, et récipiendaire de plusieurs subventions et prix de recherche, ainsi que d'un prix de supervision d'étudiants aux cycles supérieurs.

La recherche de Dre Paradis porte sur l'acquisition des langues chez l'enfant, sur le bilinguisme, et sur l'acquisition de langue seconde chez l'enfant, avec une emphase sur les enfants venant de familles d'immigrés ou de réfugiés. Sa recherche est fréquemment citée et se distingue par son volume et sa profondeur; elle comprend : trois livres édités, 16 chapitres de livre, et plus de 60 articles soumis à l'évaluation de ses pairs. Paradis a publié dans les domaines de l'éducation et de la psycholinguistique appliquée, ainsi que l'acquisition des langues. Sa recherche présente un intérêt non seulement pour les spécialistes en acquisition des langues, mais aussi pour les cliniciens tels que les orthophonistes, ainsi que les enseignants et les décideurs politiques. Paradis a effectué des recherches sur l'acquisition langagière chez les enfants bilingues en français et anglais, et avec la collaboration de ses collègues, elle a cerné la diversité des expériences bilingues en incluant des enfants venant d'une variété de milieux langagiers. En plus des populations typiques, sa recherche a examiné des populations cliniques bilingues, en particulier des enfants bilingues avec un trouble de développement du langage (appelé aussi le trouble spécifique du langage). Cette recherche a une pertinence clinique, car elle explique la variété des trajectoires bilingues. Plus récemment, elle a aussi mené des recherches sur des locuteurs bilingues avec un trouble du spectre de l'autisme. Locutrice passionnée du français et de l'anglais, son travail a modifié l'objectif de la recherche en acquisition de manière à inclure les enfants bilingues. Ceci est particulièrement pertinent dans les sociétés multilingues comme celle du Canada.

La communication des découvertes et des implications de sa recherche a été essentielle dans la carrière de Dre Paradis. Elle a organisé de nombreux ateliers et séminaires à travers le Canada pour les enseignants, les administrateurs scolaires, les orthophonistes, et les experts en éducation spécialisée portant sur plusieurs sujets liés au bilinguisme et à l'éducation bilingue. En consultation avec les partenaires communautaires, elle a créé le Centre de ressources en anglais comme langue seconde chez l'enfant, a développé des questionnaires s'adressant aux parents, comme le Questionnaire du développement langagier de l'Alberta, qui examine le développement de la langue maternelle, et le questionnaire de l'environnement langagier de l'Alberta, qui étudie des modèles de l'utilisation du langage dans les foyers bilingues. En outre, elle a participé à plusieurs entrevues à la radio et dans la presse écrite et a écrit des textes où elle défend l'approche empirique de l'étude du bilinguisme afin de réfuter les mythes et les idées préconçues. Johanne est impliquée dans la Coalition pour l'inclusion sociale (Edmonton), un groupe qui promeut l'intégration à long terme, l'inclusion sociale, et l'établissement de nouveaux arrivants, et qui traite de la question du maintien de la première langue. Plusieurs de ses projets subventionnés portent sur les meilleures pratiques et les défis dans le domaine de la langue et de l'alphabétisation parmi les immigrés et les réfugiés.

En résumé, Johanne Paradis est un exemple parfait d'une chercheuse canadienne, à la fois en termes de recherche pure et de mobilisation de connaissances. Paradis excelle également en tant que mentor et administratrice. L'Association canadienne de linguistique a le plaisir de reconnaître son service exceptionnel en décernant à Dre Paradis son Prix national d'excellence en 2020. 


\section{Dr. Johanne Paradis}

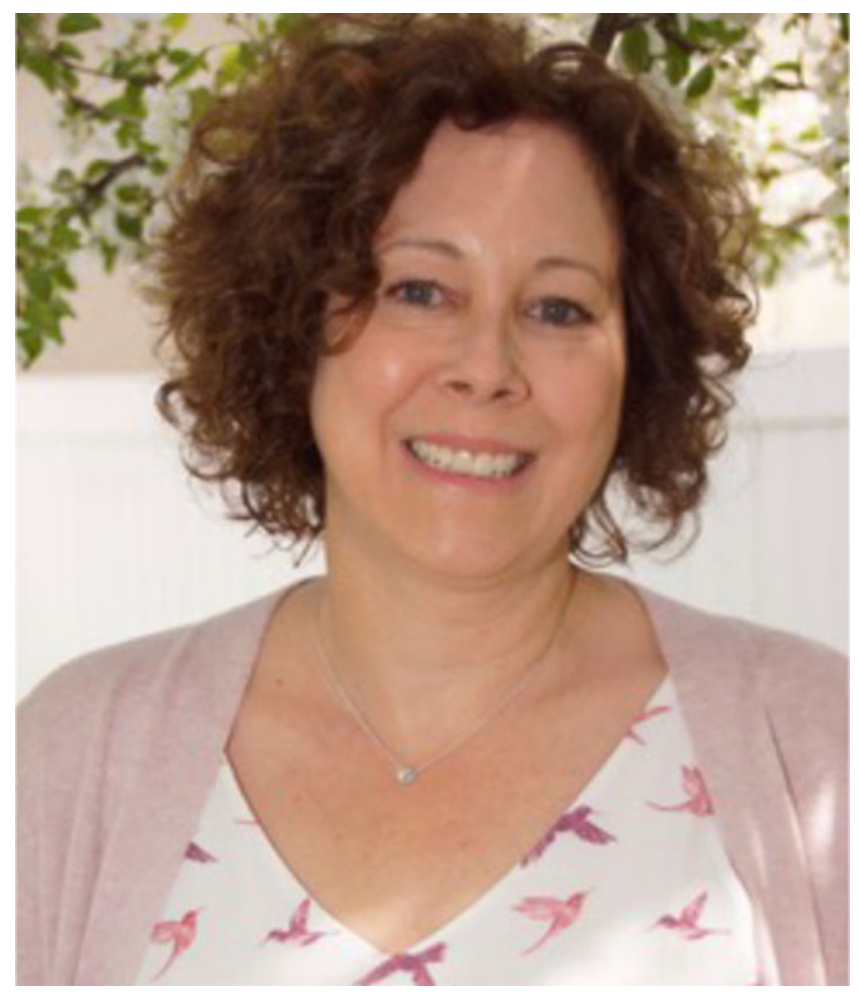

Dre Johanne Paradis 


\section{Early Career Researcher Award, Canadian Linguistic association Prix de la chercheuse en début de carrière, Association canadienne de linguistique 2020}

\section{Dr. Suzi Oliveira de LiMA}

Dr. Suzi Lima is an early career researcher who has already contributed substantially to language research, demonstrated innovation in research and dissemination, and engaged in practice and policy development in the broader community. Her theoretical focus is the pragmatics and semantics of number and quantity, and she has made contributions to formal semantics, typology, language acquisition, psychology, language documentation and revitalization, and the study of indigenous languages. She has held several research grants and has presented her work at the world's top conferences. She has published many peer-reviewed research works, and has also prepared, with indigenous communities of Brazil, a dictionary of verbs (Yudja) and a co-authored a pedagogical grammar (Kawaiwete), to be published. She is also in demand as an invited speaker and as an innovative teacher and mentor.

Dr. Lima holds a BA and MA from the University of São Paulo (Brazil), and a PhD from the University of Massachusetts. Since her 2014 graduation, she has held a post-doctoral fellowship at Harvard University and has been an Assistant Professor at the Universidade Federal do Rio de Janeiro, and in the Department of Linguistics, and the Department of Spanish and Portuguese at the University of Toronto. In 2019, she accepted her current position as Assistant Professor in the Department of Linguistics at the University of Toronto.

Dr. Lima's research focusses on pragmatics, semantics, typology, language acquisition, and documentation and revitalization. She engages in experimental fieldwork, with a focus on indigenous languages of Brazil, most prominently on Yudja and Kawaiwete. In her dissertation and much of her following work, Dr. Lima has developed ingenious protocols for uncovering the mechanisms by which languages count and measure things and substances, making novel contributions to the semantics and typology of number and quantity. Her work is also innovative in investigating the acquisition of these concepts in understudied languages, through the study of monolingual and bilingual speakers of indigenous languages and/or Brazilian Portuguese. She has also collaboratively investigated questions of general cognition, such as how mathematical reasoning relates to cultural practice.

Dr. Lima has also been exemplary in the ways she has shared her research with academic and non-academic communities. For example, her collaborative work has resulted in a questionnaire which is a masterpiece of elicitation and experimental design, targeting detailed semantic properties by using methods such as translation, production, and comprehension tasks. This questionnaire was applied by specialists who presented their results at a 2017 workshop, now in press as a special volume of Linguistic Variation. This ambitious project (co-organized with Susan Rothstein) 
involved both under-represented scholars and under-represented languages, demonstrating Dr. Lima's qualities of leadership. Dr. Lima also reaches out to communities and activists in her work, twice funded by United Nations/National Indian Foundation/Indian Museum in Brazil, to document the Kawaiwete language. This work has involved educational workshops for teachers and collaborations with community leaders and researchers, and has resulted in the production of educational materials such as a pedagogical grammar and a dictionary draft. One reading book (of Kawaiwete songs) was published in 2015 by the Museu do Índio. Dr. Lima has also collaborated in creating a database featuring resources for documentation and methods for fieldworkers.

Dr. Lima is also an inspiring teacher and mentor, demonstrated in particular by her research excursion program courses where she takes undergraduate students to Brazil for hands-on documentation and fieldwork experience (https://uoftbrazil.blog/).

In summary, Suzi Lima is an extraordinary early career researcher who has already achieved distinction in a range of areas, including theoretical, experimental, and documentation linguistics, while also demonstrating innovation in teaching and community outreach. The Canadian Linguistic Association is delighted to recognize her achievements by awarding Dr. Suzi Lima our inaugural Early Career Researcher Award in 2020.

Dre Suzi Lima est une chercheuse en début de carrière qui a déjà fait une imposante contribution à la recherche sur la langue. Suzy Lima a fait preuve d'innovation en matière de recherche et de diffusion, et s'est engagée dans la pratique et dans l'élaboration de politiques dans la communauté au sens large. L'axe principal de sa recherche touche au domaine de la pragmatique et de la sémantique du nombre et de la quantité. Lima a apporté sa contribution aux domaines de la sémantique formelle, de la typologie, de l'acquisition des langues, de la psychologie, de la documentation et la revitalisation des langues, ainsi que de l'étude des langues autochtones. Elle a obtenu plusieurs subventions de recherche et a présenté ses travaux dans les plus grandes conférences internationales. Lima a publié de nombreux travaux dans des revues avec articles évalués par des pairs; en collaboration avec les communautés autochtones du Brésil, elle a rédigé un dictionnaire (yudja) et (en tant que co-auteure) une grammaire pédagogique (en voie de publication). Elle est également recherchée comme conférencière et comme enseignante et mentor innovatrice.

Dre Lima est titulaire d'un baccalauréat et d'une maîtrise de l'Université de São Paulo (Brésil), et d'un doctorat de l'Université du Massachusetts. Depuis 2014, elle a obtenu une bourse postdoctorale à l'Université Harvard, puis des postes de professeure adjointe à l'Université Fédérale de Rio de Janeiro ainsi qu'aux départements de linguistique, puis d'espagnol et de portugais, de l'Université de Toronto. Depuis 2019, elle détient le poste de professeur adjoint au département de linguistique de l'Université de Toronto.

La recherche de Dre Lima est centrée sur la pragmatique, la sémantique, la typologie, l'acquisition des langues, la documentation et la revitalisation. Elle s'engage dans le travail de terrain avec une emphase spéciale sur les langues autochtones du Brésil, particulièrement sur le yudja et le kawaiwete. Dans sa thèse et dans ses travaux antérieurs, Dre Lima a développé des protocoles fort ingénieux afin de découvrir les 
mécanismes utilisés par les langues pour compter et mesurer des objets et des substances, en apportant des contributions importantes à la sémantique et la typologie du nombre et de la quantité. Son travail innove également dans la recherche sur l'acquisition de ces notions dans des langues peu étudiées; cette recherche a été effectuée auprès de locuteurs monolingues et bilingues de langues autochtones et du portugais du Brésil. Suzi Lima a également collaboré à des recherches sur la cognition générale, comme, par exemple, le lien entre le raisonnement mathématique et les pratiques culturelles.

Dre Lima est un modèle dans la façon dont elle partage sa recherche avec les communautés universitaire et non-universitaire. Par exemple, son travail collaboratif a mené au développement d'un questionnaire qui est admirable au chapitre de l'élicitation et de la conception expérimentale, ciblant l'étude détaillée des propriétés sémantiques à l'aide de méthodes comme la traduction, la production, et la tâche de compréhension. Ce questionnaire a été utilisé par des spécialistes qui ont présenté leurs résultats à l'atelier de 2017, et constituera un volume spécial de la revue Linguistic Variation. Ce projet ambitieux (mené conjointement avec Susan Rothstein) a impliqué des chercheurs et des langues peu représentés, mettant en lumière les qualités de direction de Dre Lima. Dre Lima maintient aussi des contacts avec des communautés et des militants dans son travail, par deux fois subventionné par les Nations Unies/ la Fondation nationale indienne/le Musée indien du Brésil, qui documente la langue kawaiwete. Ce travail comporte aussi des ateliers de formation pour les enseignants et des collaborations avec les leaders et chercheurs communautaires, et a mené à la production de matériels didactiques tels qu'une grammaire pédagogique et l'ébauche d'un dictionnaire. Un livre de lecture (de chansons kawaiwete) a été publié en 2015 par le Museu de Índio. Dre Lima a aussi collaboré à la création de la base de données offrant des ressources pour la documentation et des méthodes pour les chercheurs de terrain.

Dre Lima est aussi une enseignante et mentor motivante; ceci est démontré en particulier par son programme d'excursions de recherche : elle dirige un groupe d'étudiants du premier cycle au Brésil pour une expérience de documentation et de travail de terrain (https://uoftbrazil.blog/).

En résumé, Suzi Lima est une chercheuse débutante extraordinaire. Elle a su se démarquer dans une série de domaines, incluant la linguistique théorique et expérimentale, et dans la linguistique de la documentation, tout en faisant preuve d'innovation dans le rayonnement communautaire et éducatif. L'Association canadienne de linguistique a le plaisir de reconnaître ses réussites en décernant à Dre Suzi Lima le premier Prix de chercheur débutant en 2020. 


\section{Dr. Suzi Oliveira de Lima}

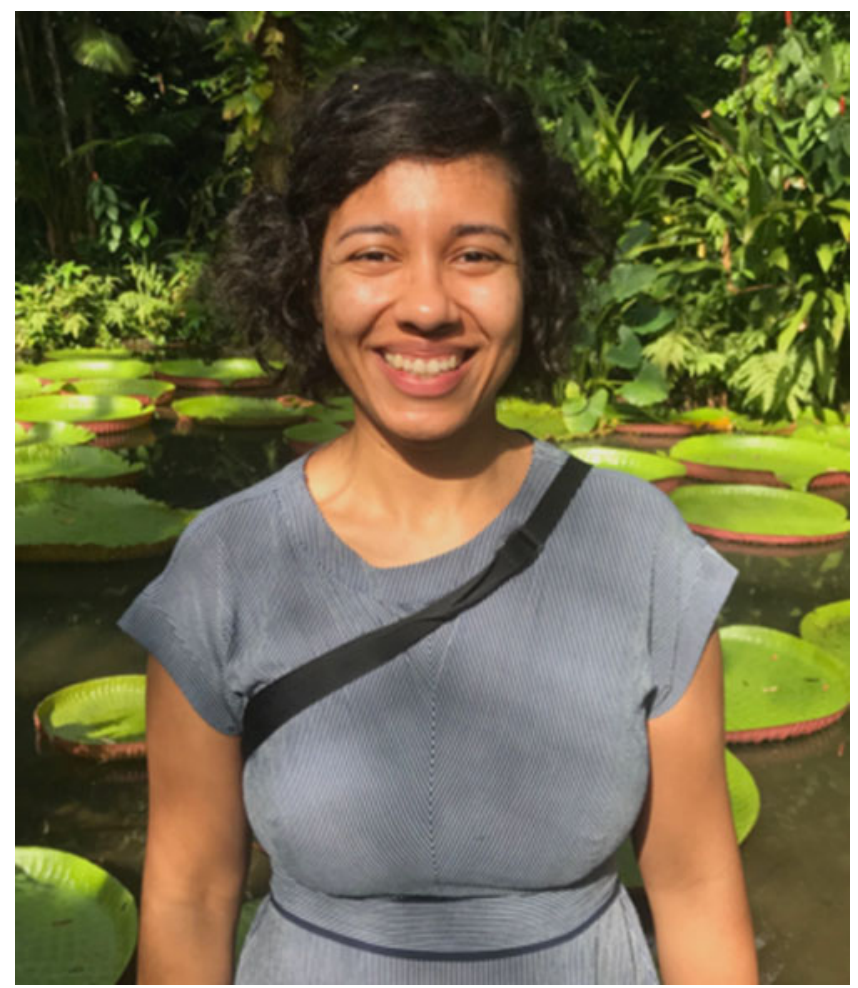

Dre Suzi Oliveira de Lima 


\title{
Student Achievement Awards Prix d'excellence étudiant CLA/ACL 2020
}

\author{
Université of Western Ontario (on line / en ligne)
}

\section{Best presentation/Meilleure communication}

Deborah Allotey (Western University): "Overt pronoun subjects of infinitival predicates in Gã"

Allotey gave an excellent talk, with solid, compelling, and clear argumentation supporting a high-quality analysis. The data were theoretically interesting and very well presented. A lively question period demonstrated that the audience was highly engaged and intrigued by the talk, and in addressing questions, Allotey demonstrated that her knowledge and understanding of the phenomenon and the theoretical questions it raises extend beyond the specific focus of the presentation.

$$
* * *
$$

Allotey a fait une excellente présentation portée par une argumentation solide, claire et incontestable soutenant une analyse de haute qualité. Les données, fort intéressantes en matière théorique, ont été fort bien exposées. Une période de questions animée a révélé que les participants étaient très engagés et intrigués par la communication. Les réponses d'Allotey aux questions des participants ont démontré que ses connaissances et sa compréhension du phénomène et des questions théoriques allaient bien au-delà du sujet central de sa présentation.

\section{Honourable mention / Mention honorable}

Una Y. Chow (University of British Columbia): "Voice Quality of Gitksan Ejectives"

\section{Meilleure affiche / Best poster}

Brittany MacDonald (University of Calgary): "What type of pronouns do Korean and Japanese have?"

MacDonald identified an interesting research question and clearly laid out the predictions of the theoretical proposal under investigation as well as the conclusions of her study. The poster was well organized and easy to follow, and also adapted well to the virtual format of the conference by providing a short recorded walkthrough of the poster's content. 
MacDonald a identifié une question de recherche intéressante et a exposé clairement les prédictions de la proposition théorique sous examen, ainsi que les conclusions de son étude. L'affiche était bien organisée, facile à suivre, et bien adaptée au format virtuel de la conférence grâce à un bref enregistrement explicitant le contenu de l'affiche. 